\title{
Self-monitoring and professional feedback through CloudRehab, a Mobile Cloud Platform for Neuro-rehabiliation
}

\author{
Gustavo Cuberos Urbano \\ Dpto. Personalidad, Evaluación y \\ Tratamiento Psicológico \\ University of Granada \\ Granada, Spain \\ gustarama@yahoo.es
}

\author{
Angel Ruiz-Zafra, Manuel \\ Noguera, Kawtar Benghazi \\ Dpto. Lenguajes y Sistemas \\ Informáticos \\ University of Granada \\ Granada, Spain \\ angelr@ugr.es, \\ mnoguera@ugr.es, benghazi@ugr.es
}

\author{
Alfonso Caracuel \\ Dpto. Psicología Evolutiva y de la \\ Educación \\ Centro de Investigación Mente \\ Cerebro Comportamiento \\ University of Granada \\ Granada, Spain \\ acaracuel@ugr.es
}

\begin{abstract}
This paper presents the development of an m-Health platform for supporting the rehabilitation process of patients with acquired brain injury. A six-step methodology for neuro-rehabilitation was supported by the platform called CloudRehab. This cloudsupported home-based rehabilitation platform includes a mobile application and a web application. The mobile application has been designed to record and play videos simultaneously, as well as collect physiological and behavioral data to be uploaded to the cloud. The collected data are accessed through a web application for professionals of health. A single subject research design indicated the platform was effective for improving a daily living activity of a patient with chronic sequelae after stroke. CloudRehab improve the performance of the rehabilitation exercise in a realcontext training, contributing to the effectiveness of the rehabilitation process. The use of technological solutions for supporting neuro-rehabilitation can seamlessly provide benefits to patients and health systems.
\end{abstract}

\section{Keywords}

Tele-rehabilitation, Neuro-rehabilitation, m-health, Acquired Brain Injury, Feedback, Self-monitoring, Cloud technology, CloudRehab

\section{INTRODUCTION}

Patients with acquired brain injury (ABI) due to traumatic brain injury or stroke undergo a variety of physical, cognitive and emotional impairments. Interdisciplinary teams expend many time in neuro-rehabilitation of these patients. Some of them must relearn even how to walk, talk and many activities of daily living. For that reason, patients have to train several times until perform these activities in a natural way. Feedback to patients about their performance of activities is a key feature in rehabilitation. Health professionals through verbal and descriptive information give feedback. However, a single professional cannot provide enough and fine feedback to patients in the more usual format in neurorehabilitation, the group training.

Permission to make digital or hard copies of all or part of this work for personal or classroom use is granted without fee provided that copies are not made or distributed for profit or commercial advantage and that copies bear this notice and the full citation on the first page. To copy otherwise, to republish, to post on servers or to redistribute to lists, requires prior specific permission and/or a fee.

REHAB 2014, May 20-23, Oldenburg, Germany

Copyright (c) 2014 ICST 978-1-63190-011-2

DOI 10.4108/icst.pervasivehealth.2014.255384
Evidence based practices indicate that patients' self-monitoring is possible in rehabilitation after ABI [1]. Self-monitoring is complementary to feedback from professionals for allowing patients to identify and discriminate between correct and incorrect components of a rehabilitation activity. Video-feedback of performance of rehabilitation tasks is especially useful for patients after ABI [2]. Video-feedback is a proper way to develop selfmonitoring. Many works have investigated the potential of the video capture technology and developed platforms that enabled users to interact with graphic objects [3]. Visual information about performance makes possible to self-correct mistakes made in the sequence of a rehabilitation activity.

Tele-rehabilitation can be helpful after ABI. Patients need intensive and long period of rehabilitation in outpatient medical centers [4]. This means a great number of displacements and time waste for patients coming from places far away from the center. In addition, many consequences of $\mathrm{ABI}$ remain as chronic sequelae after discharge from outpatient rehabilitation programs. After this time, rehabilitation at home by themselves can provide benefits to these patients. Despite the ideal training should be conducted within the patient's own real-life context [5], many patients stop training at home, and then some of the improvements and learning acquired after a hard treatment fade away. Lack of feedback from professionals during independent performance of rehabilitation exercises may influence this interruption. In this regard, mRehabilitation is emerging as an appropriate technique to enable effective treatment of patients in their natural environments. Previous proposals have mainly consisted in several forms of video tracking of patients by using different types of devices and technologies, such as video recording or videophones [6]. In contexts where video is extensively used, the management and labeling of recorded material is also a crucial issue, however, none of them seem to provide this kind of support or make use of the services and functionalities provided by mobile devices alongside cloud computing [7], for subsequent reference, collaboration and discussion between professionals, and with caregivers, at anytime, from anywhere [8].

This paper summarized the project for developing a m-Health cloud-based platform for supporting the neuro-rehabilitation process of patients after acquired brain injury. 


\section{THE REHABILITATION PROCESS SUPPORTED BY CloudRehab}

\subsection{Case description}

The participant was a 53 years old male with right hemiplegia after a stroke 10 months ago. The main disability was the mobility of the right upper limb and right lower limb, causing limitations in finger extension and flexion. His body awareness and spatial competence were normal and there were no limitations in understanding the information given. Most of his activities of daily living (ADLs) were accomplished by compensating with the left arm

\subsection{Research Design}

A single-subject design was used to evaluate the pre (Baseline) and post (CloudRehab) training phase, following the rating scale method for the quality of single-case experimental designs and tests $\mathrm{n}-1$ : ROBINT. Buttoning and unbuttoning the shirt using both upper limbs was the selected monitoring task.

Scores were obtained after each recording based on predefined criteria for each behavior object. The implementation for each criterion of each behavior was rated from 0 to 4 (4: Very good execution; 3: Good execution, 2: Poor execution; 1: Very poor execution and 0 : unexcuted). These assessments were performed by two blinded therapists. The videos, both base line and intervention, are provided in the order given by a list of random numbers.

\subsection{Procedure}

The project started with a summary of those needs of patients and professionals about neuro-rehabilitation that should be addressed from $m$-health. These needs were basically summarized as follows: (1) professional had to train patients on how to perform properly each activity, (2) patients had to memorize and remember how to do it every single time they must train with the presence of the professional, and (3) without the presence of the professional, (4) patients had to get behavioral and emotional feedback during and after their performance, and (5) professional and patients need to communicate quickly and efficiently, and this included the recording of data. From these needs, we determined which steps should compose the m-health tele-rehabilitation process.

\subsubsection{Training of patient at the medical center}

At the beginning, as in any rehabilitation program, the patient should be trained by the corresponding professional in the health center. This is a teaching-learning process adapted to patient characteristics. The training exercise must be divided in its sequential components. Close supervision and guidance of professional helps patient to perform the target exercise in an appropriate way.

\subsubsection{Recording the performance of the rehabilitation exercise \\ Once the patient has learned to perform the training task, the professional records a guided execution with a smartphone or other record device. This so called "self-tutorial video" is to be uploaded to the system in order to be available for patient later on. A web- based video manager helps professionals to divide the file with the recorded exercise in several relevant steps. The establishment of sequential phases into the exercise supports learning and performance of patients, who can watch their own recorded tutorial videos in a step-by-step basis.}

\subsubsection{Training of patient at home}

Patients can perform a target rehabilitation exercise at home, without direct professional supervision, but using a video tutorial as guidance. At the beginning of a home-based performance of rehabilitation activities, it is advisable that patients watch the selftutorial video first and then try to reproduce it in the same way that they did at hospital. Of course, patient performance during training at home should be also recorded with him/her device, and automatically uploaded to the system for asynchronous supervision. Patients encouraged to watch their own "video of training at home" immediately get a useful feedback for self-monitoring their performance. This feedback process is also supported by reviewing and comparing their current performance recorded at home with the self-tutorial video. Subsequently, patients could try it again and correct some mistakes. The $\mathrm{m}$-health system must provide patients with a guide about when and how many times they should perform each rehabilitation exercise.

\subsubsection{Heart rate monitoring}

While patients perform rehabilitation exercises at home, they can make mistakes or get nervous for several reasons. Changes in heart rate helps patient and professionals be aware of emotional distress. For that reason, heart rate is monitored during the execution of some training activities, by a chest strap (Polar Wearlink+® Smart Bluetooth) The system should also provide some support when needed. In these cases, system may ask patient to select between a menu of possible support options. He/she could get some help for relaxing, stop and rest for a short time and/or simply continue the exercise in case of false alarms.

\subsubsection{Therapeutic session programmer}

In order to facilitate the planning of rehabilitation at home, the system allows the professionals to schedule the therapeutic sessions with specific dates, start time and end time. This promotes the realization of an adequate number of repetitions at the most convenient times of the day to be carried out.

\subsubsection{Collect and sharing rehabilitation process data. Communication between professionals and patients}

The m-health system might collect several set of data during the above described home-based rehabilitation process. Supervision of professionals may be based on videos of training, records of heart rate values, time of performances, kind of support options chosen for patients, and so on. It could be a very useful communication between patients and professional through text messages into the system. Professional could review this wide range of information at any time and provide specific feedback about patient's performance of the rehabilitation exercise he/she prescribed (how to improve or correct mistakes). They can try to motivate patients or give advice about how the rehabilitation process is going, etc.

\subsection{The application for supporting the neuro- rehabilitation process}

In order to meet all identified needs and follow the above steps, we developed a platform with two main applications:

\subsubsection{Web Application}

The web application can be used by any user of the system (health professionals, patients and their relatives). Although all of them use the same web application, each type of user (i.e., health professional, caregiver, and patient) has its own interface and restricted access to different sets of functionalities. The different functionalities provided are, among others, the management of patient information (medical and personal information), the 
management of the sessions performed by patients, review information generated by patients or real-time monitoring.

\subsubsection{Mobile Application}

The mobile application allows the training session of an exercise to be played and recorded while obtaining heart rate using a chest strap sensor to monitor patient's stress level. The application divides the device screen into two different parts. The upper part shows the images captured by the frontal camera, while the bottom part of the screen displays the training video previously recorded with the supervision of a therapist (Figure 1). This provides the patient with a useful real-time feedback about how to correctly perform the exercise and how he/she is approaching to the intended model. If the application detects that the heart rate signal of the patient has reached the threshold defined by the professionals, the application plays an information sound and shows a pop-up dialog with different options (i.e., review the video session by steps, play custom help audio or view relaxing images) in order to aid the patient to reduce their stress level. The user interface has been designed according to the criteria of health professionals in order to ensure the usability for this set of users, and make it easy to use and intuitive for the patient as well.

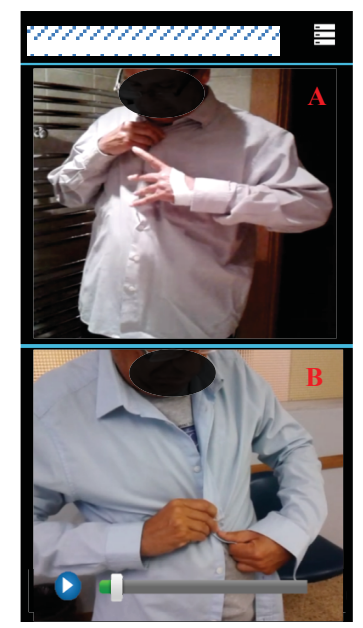

Figure 1. Self-Monitoring. Part A of screen: recording and watching current patient's performance; part B: watching self-Tutorial video

\section{RESULTS OF EFFECTIVENESS}

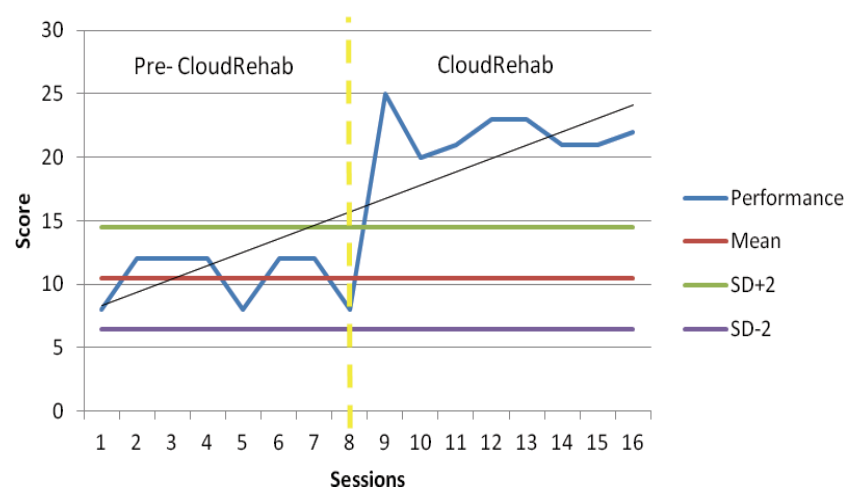

Figure 2. Total score of everyday participant' performance in baseline (Pre-CloudRehab) and treatment phases (CloudRehab)
To study the effectiveness of CloudRehab platform a single case study design with two phases was conducted. During the baseline phase ( 8 days) the participant was asked to record himself while doing the target behaviour (wear a shirt and buttoning using both upper limbs) the best he could do. Subsequently, in the treatment phase ( 8 days), the participant performed and recorded the behaviour using the CloudRehab mobile application (frontal camera). For data analysis, a split-middle trend line was applied to extrapolate data trend in the baseline phase in order to determine if the trend continues in the treatment phase [9]. Data of baseline phase was divided in half [10] by a vertical dotted yellow line (see Figure 2). The median of the data in each half of the baseline was 8 and 9 respectively. Each half of the phase was halved by the vertical green line so that it was segmented into quarters. The value of the first median (eight) was plotted on the firstquarter green line; the second median (nine) was plotted on the third-quarter line. A scarlet line throughout the baseline phase was drawn through the two median data points. An equal number of data points appeared on either side of the split-middle trend line. The line was extrapolated into the treatment phase. Seven of the eight data of the treatment $(87.5$ percent $)$ were over the split-middle trend line.

\section{DISCUSSION}

This analysis supported an improvement of patient's performance in the target behavior. Looking at features of the systems, we hypothesize there are some key factors in CloudRehab for explain its effectiveness. First, the system is a constant source of feedback to the patient, a powerful issue in rehabilitation process [11]. The self-tutorial video refreshes all what patients have to keep in mind for doing the target exercise in a right way. Then, they can review their own performance and get self-monitoring about the degree of similarity between what they did and what they should do. Availability of information in a permanent format such as videos has been successfully used in rehabilitation after acquired brain injury [1]. Thus, patients can train at home independently without too much memory requirements to remember what, when and how to do. The use of videos allows to workaround memory and executive dysfunctions, one of the most frequent sequelae after brain injury [12]. Second, training daily living activities outside the medical center, at the real-life context, promotes generalization [13]. Caregivers play a key role helping patients and professional to choose feasible activities for home-based rehabilitation. It seems reasonable that a motivated patient who is provided of professional help to practice as much as he/she want, can make larger advances in their own recovery.

\section{CONCLUSIONS AND FUTURE WORK}

The aim of this research was apply a m-Health cloud-based platform for supporting the neuro-rehabilitation process of patients after acquired brain injury. CloudRehab facilitates rehabilitation by support learning and training of rehabilitation target activities together with reducing the number of displacements of patients and caregivers to a medical center. Results from a single subject research design shows the effectiveness of this methodology for a relevant daily living activity such as dress a shirt independently. As future work we are currently working in the development of new functionalities needed for specific professional in the interdisciplinary rehabilitation team such as Speech Therapists and Nurses. On the other hand it is necessary to include more patients. 


\section{ACKNOWLEDGEMENTS}

This research work has been partially funded by the Health Office of the Andalusian Government (PI-0776-2013), the Spanish Ministry of Economy and Competitiveness with European Regional Development Funds (FEDER) under the research project TIN2012-38600 and by the Granada Excellence Network of Innovation Laboratories (GENIL) under project PYR-2014-5. The authors would also like to acknowledge contribution from COST Action AAPELE IC1303.

\section{REFERENCES}

[1] J. Malec, Training the brain-injured client in behavioral selfmanagement skills, Behavioral assessment and rehabilitation of the traumatically braindamaged (1984) 121-149.

[2] R. Brindley, A. Bateman, F. Gracey, Exploration of use of sensecam to support autobiographical memory retrieval within a cognitive-behavioural therapeutic intervention following acquired brain injury, Memory 19 (7) (2011) 745-757.

[3] P. L. Weiss, D. Rand, N. Katz, R. Kizony, Video capture virtual reality as a flexible and effective rehabilitation tool, Journal of neuroengineering and rehabilitation 1 (1) (2004) 12.

[4] L. Turner-Stokes, D. Wade, Rehabilitation following acquired brain injury: Concise guidance, Clinical Medicine 4 (1) (2004) $61-65$.

[5] M. Ylvisaker, Context-sensitive cognitive rehabilitation after brain injury: Theory and practice, Brain Impairment 4 (01) (2003) $1-16$.
[6] M. McCue, A. Fairman, M. Pramuka, Enhancing quality of life through telerehabilitation, Physical medicine and rehabilitation clinics of North America 21 (1) (2010) 195-205.

[7] A. Ruiz-Zafra, M. Noguera, K. Benghazi, J. L. Garrido, G. C. Urbano, A. Caracuel, A mobile cloud-supported erehabilitation platform for brain-injured patients.

[8] [8] A. Fox, R. Griffith, A. Joseph, R. Katz, A. Konwinski, G. Lee, D. Patterson, A. Rabkin, I. Stoica, Above the clouds: A berkeley view of cloud computing, Dept. Electrical Eng. and Comput. Sciences, University of California, Berkeley, Rep. UCB/EECS 28 .

[9] M. Perdices, R. L. Tate, Single-subject designs as a tool for evidencebased clinical practice: Are they unrecognised and undervalued?, Neuropsychological rehabilitation 19 (6) (2009) 904-927.

[10] R. R. Robey, M. C. Schultz, A. B. Crawford, C. A. Sinner, Review: Single-subject clinical-outcome research: designs, data, effect sizes, and analyses, Aphasiology 13 (6) (1999) 445-473.

[11] M. Barnes, D. Good, Neurorehabilitation approaches to facilitate motor recovery, Neurological Rehabilitation: Handbook of Clinical Neurology 110 (2013) 161.

[12] S. Dikmen, J. Machamer, J. R. Fann, N. R. Temkin, Rates of symptom reporting following traumatic brain injury, Journal of the International Neuropsychological Society 16 (3) (2010) 401.

[13] C. L. Yantz, D. Johnson-Greene, C. Higginson, L. Emmerson, Functional cooking skills and neuropsychological functioning in patients with stroke: An ecological validity study, Neuropsychological rehabilitation 20 (5) (2010) 725-738. 